\title{
Early cycle of matter in active region of star formation, and AGN objects
}

\author{
Matveenko L.I., , Sivakon S.S., Seleznev S.V. \\ Institute for Space Research \\ 117997, 84/32 Profsoyuznaya Str, Moscow, Russia \\ E-mail: Imatveenko@gmail.com
}

Jorstad S.G.

V.V. Sobolev Astronomical Institute, St. Petersburg State University,

198504, 28 Universitetskii, Petrodvorets, St. Peterburg, Russia;

Institute for Astrophysical Research Boston University

One Silber Way, Boston, MA 02215, USA

Marsher A.P.

Institute for Astrophysical Research Boston University

One Silber Way, Boston, MA 02215, USA

Graham D.A.

Max Planck Institute for Radio Astronimy

Auf dem Hügel 69, 53121 Bonn, Germany

Diamond P.J.

Jodrell Bank Observatory, The University of Manchester

Macclesfield, Cheshire, SK11 9DL, United Kingdom

\section{Abstract}

It is shown that the fine structure of the star formation region in Orion KL consists of a rigid body rotation disk and a bipolar outflow. This structure appears to be an anti-centrifuge which is accreting the surrounding neutral gas, moving it along the arms towards the centre and ejecting the rotating bipolar outflow. The outflow interacts with surrounding matter which causes an exponential growth of velocity and collimates the outflow. Reactive force of the outflow determines the precession and conical helical structure with increasing step of the jet. The counter motion of the matter in the centre stimulates the formation of a massive central object. The mass of the central body is $\mathrm{M}<0.01 \mathrm{M}$. which corresponds to an early stage of star formation. The same kinematics and structure are observed in the AGN objects, NGC 4258, $1803+784$, and M87. A counter-jet has been discovered in M87. The jet and the counter-jet have helical structures. Currents and magnetic fields are excited in the ionized medium, these in turn accelerate the processes. A low-velocity outflow is ejected by the outer part of the disk, and a relativistic high-velocity outflow by the centre part. The relativistic jet of $1803+784$ is accelerated until it reaches $v=15 \mathrm{c}$ in active zone length $\sim 5 \mathrm{pc}$.

10th European VLBI Network Symposium and EVN Users Meeting: VLBI and the new generation of radio arrays

Manchester, $U K$

September 20-24, 2010

\footnotetext{
* Speaker
} 


\section{Introduction}

Images of astronomical objects contain a great deal of information about their nature and the physical processes within them, and the information density of these images increases with their angular resolution. Our studies of the fine structure of astronomical objects such as active star formation regions in Orion KL and AGN objects show that they have an identical structure: an accretion disk and a bipolar outflow. VLBI methods have opened unlimited possibilities for theses types of studies [4]. Additionally the presence of strong $\mathrm{H}_{2} \mathrm{O}$ maser emission in some sources has further increased the level of obtainable information by offering the possibility to detect and determine the super-fine $3 \mathrm{D}$ structure. In this paper we consider the results of studies of some of these objects.

\section{Star formation region in Orion KL}

The active process of star formation is accompanied by $\mathrm{H}_{2} \mathrm{O}$ maser emission. In Orion KL we observed strong outbursts of mega-maser emission, the linear velocity of which was $v=7.65 \mathrm{~km} / \mathrm{s}$, and line width $\Delta v \approx 0.45 \mathrm{~km} / \mathrm{s}$. The structure of the active region consists of the $\sim 27 \mathrm{AU}$ disk and the bipolar outflow. The edge-on disc appears Sshaped, Fig. 1a. The tangential directions of its arms correspond to a chain of four groups of compact components, down-left. The diameter of the rings corresponds to the logarithm of brightness temperature $\mathrm{T}_{\mathrm{b}}$. The velocities of the central part of the disk correspond to the rigid body rotation $v_{\text {rot }}=\Omega \mathrm{R}$ with period $\sim 170 \mathrm{yrs}$, down-right. The rotation velocity of the outer part of the disk at $R \geq 7 \mathrm{AU}$ is $v \approx 1 \mathrm{~km} / \mathrm{s}$, which corresponds to a mass of the central body $M<0.01 \mathrm{M}_{\odot}$ in the Keplerian approximation $[5]$.

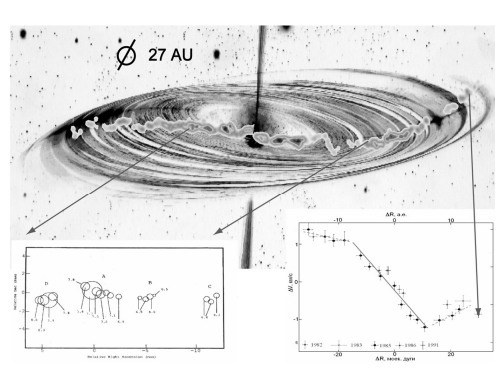

a



b

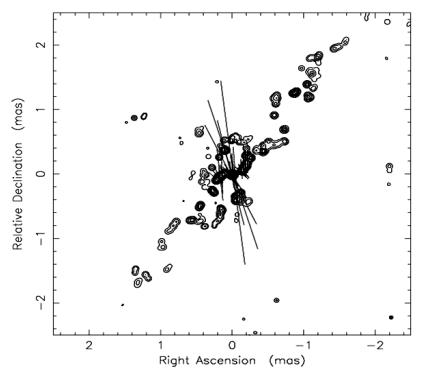

c

Fig.1. (a) The S-shape disk seen edge-on. (Lower-left) The chain of four groups of compact components. (Lower-right) The line-of-sight velocity of components versus distance. (b) The bipolar outflow. (c) The fine structure of the ejector region in polarized emission. 
The bipolar outflow has a spiral structure with an increasing step that determined by the precession of ejector axis (Fig.1b, c). Fragments of bipolar outflow are observed at distance up to $80 \mathrm{AU}$, however the outflows cross-section does not change $(\sim 0.01$ AU) implying high levels of collimation. The velocities of the $\mathrm{H}_{2} \mathrm{O}$ knots increases from $v_{o} \leq 5 \mathrm{kms}^{-1}$ to $40 \mathrm{kms}^{-1}$ at a distance of $\sim 1 \mathrm{AU}$ [4]. The surrounding matter amplifies the radiation by more than two orders at $v_{\mathrm{LSR}}=7.65 \mathrm{kms}^{-1}$. The polarization of maser emission is $\mathrm{m} \geq 50 \%$ (Fig.1c). Extraordinary changes of polarization orientation are determined by different orientation of polarization and radial velocities of the outflows at the nozzle outputs. The distance between nozzles is $\leq 0.004$ AU (Fig. 1c). The extra high level of polarization is determined by the directivity of pumping, which is due to the spin alignment of $\mathrm{H}_{2} \mathrm{O}$ molecules.

The rigid body rotating disk and highly collimated bipolar outflow are analogue of a tornado (Fig. 2). The whirlwind in the molecular cloud of Orion KL contains matter, including ice granules, which move along the arms to the nozzles and is ejected as rigid body rotated outflows.

The counter motion of the matter at the centre of the disk stimulates the formation of a central object - protostar. The gravitational field of this central object both accelerates and stabilizes the system forming a vortex. Interaction with the surrounding matter accelerates and collimates the outflows [1]. The duration of the activity is determined by the matter accumulated in the disk.

\section{Galaxy: - NGC 4258}

The same structure and kinematics are observed in the galaxy NGC 4258. Two groups of $\mathrm{H}_{2} \mathrm{O}$ maser sources are distributed in outer part of an S-shaped accretion disk $R=(0.13-0.25)$ pc, which is nearly edge-on (Fig. $2 \mathrm{a}, \mathrm{b})$ [9]. Their velocities correspond to Keplerian motion around a black-hole of mass of $\mathrm{M}=3.6 \times 10^{7} \mathrm{M}_{\odot}$. We believe that the velocities of the central group of masers varies linearly $\mathrm{d} v / \mathrm{d} R=8.3 \times 10^{3} \mathrm{kms}^{-1} \mathrm{pc}^{-1}$, corresponding to a rigid-body rotation disk $R \approx 4.5$ mas or $\sim 0.16 \mathrm{pc}$. The brightest masers are located on the inner part of the disk, $R \approx 0.35$ mas or $0.012 \mathrm{pc}$, and correspond to the tangential directions of the streams of neutral gas, which are flowing along the arms to the nozzle. Period of rigid body rotation is equal to $\sim 740 \mathrm{yrs}$. 

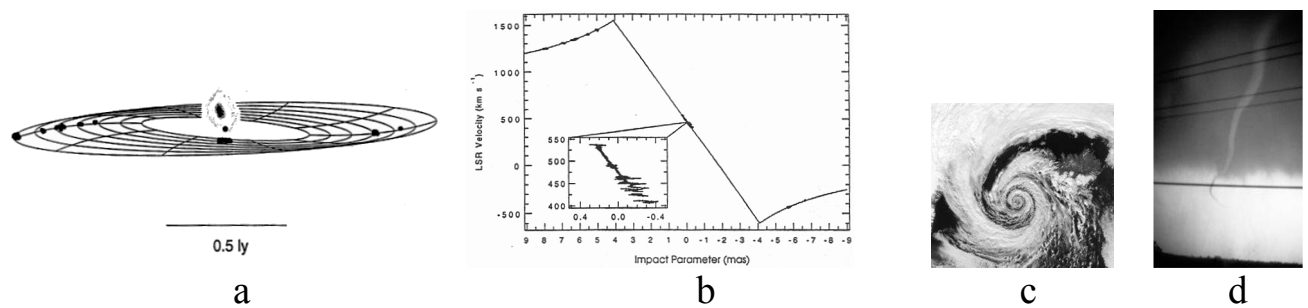

Fig.2 . (a) The distribution of the $\mathrm{H}_{2} \mathrm{O}$ maser sources in NGC 4258. (b) The line-of-sight velocity versus the distance. (c) A tornado: vortex, (d) a tornado: outflow.

\section{BL Lac object $1803+784$}

The jet of BL Lac object $1803+784$ has a helical structure with increasing step and curved axis [6]. The core is surrounded by an ionized medium, which absorbs synchrotron radio emission and determines cut off spectrum of the knots. The medium is transparent at millimeter wavelengths, and the ejector and nearest part of the jet are visible at $\lambda=7 \mathrm{~mm}$. The ejector (size $\approx 0.06 \mathrm{pc}$ ) is surrounded by a ring structure, the diameter of which is $\sim 1.4 \mathrm{pc}$ and the ring width is $0.25 \mathrm{pc}$ (Fig. $3 \mathrm{a}, \mathrm{b}$ ). Size of the disk is similar to NGC 4258.

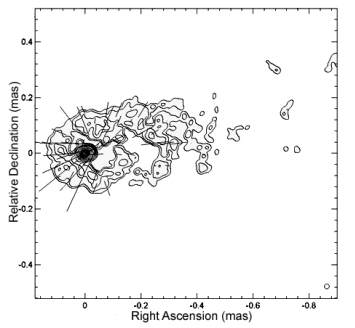

a

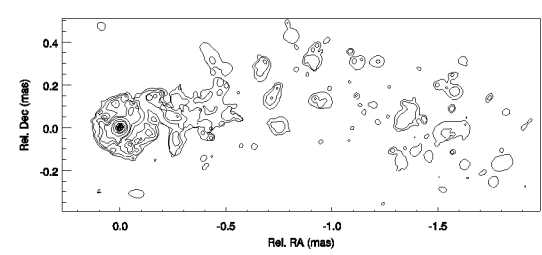

b

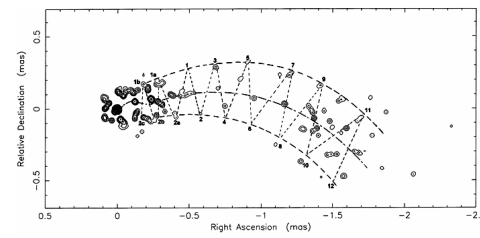

$\mathrm{c}$

Fig. 3. (a) The ejector region in polarized emission at $\lambda=7 \mathrm{~mm}$, angular resolution is $50 \mu$ as. The ejector and nearest part of the jet, are shown in (b) at a resolution of $50 \mu \mathrm{as}$, and (c) at a resolution $20 \mu$ as.

The outflow of relativistic plasma ejected nearly in the direction of the observer, and then turns to the SW. The outflow enters the active zone, the $5 \mathrm{pc}$ long, and is accelerated until the visible velocity $15 \mathrm{c}$. High-energy relativistic electrons after their escape from the active zone are visible at a distance of $\sim 1$ mas or $6 \mathrm{pc}$, which corresponds to a lifetime $\mathrm{t} \geq 18 \mathrm{yr}$. The jet is surrounded by the short-step helical structure (Fig. 3b, 3c). The polarization of the outflow at the exit of the nozzle reaches $30 \%$. Polarization of the disk fragments is $4-11 \%$ and has a radial orientation, which suggests a ring magnetic field in the disk (Fig 3a). The orientation of the polarization of the high-velocity outflow aligns with the velocity vector and the magnetic force tube, which additionally increases the gas-dynamics collimation [6]. 


\section{The Radio galaxy: M 87}

We studied structure of the radio galaxy M87 at wavelengths $0.7-18 \mathrm{~cm}$. A compact bright radio source - ejector and the bipolar outflow or its fragments are visible at all frequencies. The ejector is located in the central part of S-shaped structure - the accretion disk, which is visible nearly edge-on. This is shown at wavelength $\lambda=2$ $\mathrm{cm}$ with angular resolution 0.1 mas (Fig. 4a). The diameter of the visible part of the disk is equal 1.5 mas or $0.12 \mathrm{pc}$, with a thickness of $0.015 \mathrm{pc}$. A counter-jet, which is the mirror image of the near-side jet can also be seen [8]. The brightest visible parts of the jet and counter-jet are proportional $\rho(\lambda) \approx 0.036 \lambda$ pc, where $\lambda$ in $\mathrm{cm}$. However, the jet has long post-emission that is visible at large distance. At decimeter wavelengths the post-emission is observed up to $\sim 40 \mathrm{pc}$. The high-velocity bipolar outflow is surrounded by non-relativistic plasma. The knot velocities are $v \leq 0.02 \mathrm{c}$. The reactive force of the ejected outflow determines multimode precession - helical structures with increasing steps and the curved axis.

The non-relativistic plasma of the counter-jet is visible in $\mathrm{H} \alpha$ lines up to $\sim 1 \mathrm{kpc}$ like two parallel streams. We propose that the streams are the tangential directions of the hollow outflow - tube. The magnetic field is parallel to the jet. The jet splits into two parallel streams at a distance of $\sim 40$ mas or $\sim 3 \mathrm{pc}, \lambda=2 \mathrm{~cm}$, and is visible out to $\sim 8$ pc. The split is the result of reduction in emission of the relativistic jet.


Fig. 4. (left) The fine structure of the core region of M87 at $\lambda=2 \mathrm{~cm}$, resolution 0.1 mas (upper part). The core region, resolution $50 \mu$ as at $\lambda=7 \mathrm{~mm}$ (lower panel). (right) The core region at $\lambda=7 \mathrm{~mm}$, resolution $0.2 \times 0.4$ mas [3]. Knots of the high-velocity outflow are marked as red \#, and the low-velocity components as yellow stars.

The both structures are the helix with variable step. The two chains of the nonrelativistic features at $\lambda=7 \mathrm{~mm}$, marked by yellow stars, are located parallel to the relativistic jet, marked by red \# (Fig. 4,b) [3]. We propose that this is tangential directions of helix of the low-velocity jet. The different steps of the helix are determined by the outflow velocities. The diameter of the high-velocity outflow is increasing, and united with the wall of the low-velocity outflow at a distance $\rho \geq 10$ mas or $\geq 0.8$ pc. The 
low-velocity outflow is ejected from outer part of the disk and the high-velocity outflow from the central part of the disk [8].

\section{Conclusions}

Star-formation regions and AGN are objects of different nature, but they have similarities in their kinematics and structures: the rigid body rotating disk and the high collimated bipolar outflow - the structure of the whirlwind. In each case the disk absorbs surrounding matter and ejects matter in a rigid body rotating outflow. An external part of the outflow interacts with the surrounding matter that exponentially intensifies the velocities of the movement and the rotation of the high collimated outflow. Ejection outflows create multimode precession which determines a helical structure with a curved axis and variable step which is often observed. An object of large mass is formed in the central part of the disk, the gravitational field of which accelerates the process of formation of the system. In the case of the star-formation region in Orion KL this is neutral matter, but for AGN objects it is plasma. The currents and magnetic fields are excited in the ionized medium, additionally accelerating the activity of processes and stabilizing the system $[1,2]$. The gas-dynamic instability of the stream determines the precession and the helical structure with an increasing step. The outer region of the jet is determined by the magneto-accelerated wind and the thickness by high-velocity flow of relativistic plasma.

\section{Acknowledgments}

These studies was supported by the Program of the Presidium of the Russian Academy of Sciences "Origin and evolution of Stars and Galaxies", and the Russian Foundation for Basic Research (project No 08-02-00154).

\section{References}

[1] M.G.Abramjan, An evolution of astrophysical flows generated by whirlwind, Astrophysica 52, 135 (2009).

[2] R.V.E.Lovelace, and M.M.Romanova, Relativistic Poynting Jets from Accretion Disks, Astroph.J., 2003, 596, L159.

[3] C.Ly, R.C.Walker, J.M.Wrobel, High-frequency VLBI Imaging of the Jet Base of M87, Astroph.J., 660, 200, 2007.

[4] L .I. Matveyenko, N.S.Kardashev, and G.B.Sholomitski, On interferometer with big baseline, Radiophisica , 4, 651, 1965.

[5] L.I. Matveyenko, S.S. Sivakon, Polarized Emission from the Ejector in Orion KL, Astronomy Letterts, 2008, 34, 12, 820-831. 
[6] L.I. Matveyenko, S.S. Sivakon, S.J. Jorstad, A.P. Marscher, Structural Peculiarities of the AGN Object 1803+784, Astronomy Letterts, 2010, 36, 3, 151-166.

[7] L.I. Matveyenko, V.A. Demichev, Bipolar Outflow in Active Region Orion KL, Astronomical Review, 2010, 54, 11, 986-994.

[8] L.I. Matveyenko, S.V., Seleznev, Fine Structure of Galaxy M 87 Core-Jet, Astronomy Letterts, 2011,37, 3 .

[9] M.Miyoshi, J.Moran, J.Herrnstein, et al. Evidence for a Massive Black Hole from High Rotation Velocities in a Sub-Parsec Region of NGC 4258, Nature, 373, 6510, 127, (1955). 